\title{
Autoimmune Hemolytic Anemia:Focusing on Therapy According to Classification
}

\author{
Fergun Yilmaz ${ }^{1 *}$ and Filiz Vural ${ }^{2}$ \\ ${ }^{1}$ Izmir Katip Celebi University, Ataturk Training and Research Hospital, Karabaglar Izmir,Turkey \\ ${ }^{2}$ Ege University Medical School, Department of Hematology, Bornova Izmir, Turkey
}

Received: January 21, 2017; Accepted: February 01, 2017; Published: March 09, 2017

*Corresponding author: Fergun Yilmaz, MD, Izmir Katip Celebi University, Ataturk Training and Research Hospital Izmir Turkey, E-mail: fergunaydin@hotmail.com

\begin{abstract}
Autolmmune Hemolytic Anemia (AIHA) is a rare disease characterized by premature destruction of the red blood cells due to autoantibodies produced against erythrocyte antigens. Early destruction shortens the lifespan of the erythrocytes and causes anemia, the hallmark of the disease. It is a heterogeneous disease so the clinical presentation is diverse from a mild asymptomatic anemia to a refractory disease associated with mortality and morbidity. The different classifications are constituted to determine the prognosis and appropriate treatment strategy. It can be classified according the absence or presence of an underlying cause as primary or secondary. Another classification in AIHA is according to the type and thermal activity of the antibody as Cold Agglutinin Disease (CAD) or warm AIHA. In CAD, rituximab monotherapy or combinations is the treatment of choice. In the warm AIHA, the steroid should be preferred as a first line therapy. In the steroid refractory or relapsed patients, rituximab or splenectomy can be chosen according to the patient's characteristics and the experience of the doctor. Since treatment mainly depends on the subtype of the disease differential diagnosis between the subtypes becomes important. This review will be focused on the classification and the treatment of AIHAs according to subtypes.
\end{abstract}

Keywords: Autoimmune hemolytic anemia; Classification; Treatment

\section{Introduction}

Normal lifespan of erythrocytes are approximately 120 days Hemolysis is defined as decreased lifespan of the erythrocytes due to early destruction. Hemolytic anemia is the condition in which hemolysis cannot be compensated by increased erythropoiesis and uncompensated hemolysis results in anemia. In the AutoImmune Hemolytic Anemia (AIHA) premature destruction of the red blood cells is due to autoantibodies produced against erythrocyte self-antigens. It is a rare disease with an incidence of one to three per 100.000 people annually. AIHA is a heterogeneous disease with a diverse clinical presentation from asymptomatic compensated hemolysis without anemia to fulminant disease associated with mortality and morbidity $[1,2]$. For determining prognosis and appropriate treatment modality, different classifications are performed. The disease can be classified according to the place of hemolysis as intravascular or extravascular. There are also other classifications according to underlying cause and type and thermal activity of the existing antibody as primary or secondary and warm or cold respectively. In this review, we focused on the treatment of AIHA according to classification.

\section{Classification}

\section{According to underlying cause; primary or secondary}

AIHAs can be subdivided according to underlying disease (Table 1). No underlying disease or agent can be distinguished in primary AIHA, whereas in the secondary cases, an associated condition is well documented such as autoimmune diseases, malignancies, infections or drugs. Malignancies are one of the most common causes. AIHA can be seen at the diagnosis or during the course of cancers such as lymphomas, Chronic Lymphocytic Leukemia (CLL), and solid tumors. AIHA can be also associated with autoimmune diseases particularly Systemic Lupus Erythematosus (SLE). Other examples of autoimmune diseases are antiphospholipid syndrome, Sjogren's syndrome, and rheumatoid arthritis [3-7]. Beside these, many drugs can cause AIHAs [8,9].

Secondary AIHAs are more challenging since the diagnosis and the treatment of the underlying cause is also necessary along with hemolysis. So the underlying causative agent and disease should be searched in details.

\section{According to the thermal activity of the antibody: warm, cold or mixed}

AIHA can also be classified as cold, warm or mixed according to type and optimal reactivity temperature of the auto-antibodies (Table 1). Warm AIHA (WAIHA) accounts for $80 \%$ of all AIHAs in adults. It is characterized by IgG type auto-antibodies that react with red blood cell antigens optimally at $37^{\circ} \mathrm{C}$. The destruction of antibody coated red blood cells occurred at reticuloendothelial system especially in the spleen which is called extracellular hemolysis $[10,11]$. As mentioned before, it can be subdivided as primary or secondary. In the primary or idiopathic form no 
Table 1: The classification of AIHA

A. Warm reactive antibodies

1.Primary or idiopathic warm AIHA

2. Secondary warm AIHA

a. lypmhoproliferative disease

b. autoimmune diseases and rhemotologic diseases

c.drugs

d. solid tumors

B. Cold reactive antibodies

1.Cold agglutinin disease

a. primary or idiopathic

b. secondary

i. infections

ii. lypmphoproliferative diseases

2. Proxysmal cold hemoglobinuria

C. Mixed type :associated with both warm and cold reactive antibodies

1. Primary

2. Secondary

AIHA : autoimmune hemolytic anemia

underlying pathology can be recognized. Secondary wAIHAs may be associated with variety of underlying conditions. Secondary forms accounts for $50 \%$ of the wAIHA cases.

Cold Agglutinin Disease (CAD) and Paroxysmal Cold Hemoglobinuria(PCH) are caused by cold reactive antibodies. CAD is mostly observed in middle aged or elderly patients with slight female predominance [12]. It accounts for $15 \%$ of all AIHAs [13]. CAD is mostly caused by IgM type autoantibodies that react with the red blood cell antigens at low temperatures. Primary form of the disease is characterized by clonal B cell proliferation in the bone marrow and IgM type monoclonal gamapathy [12]. Secondary CAD is typically due to a lymphoproliferative diseases such as lymphoma and CLL, or infections such as infectious mononucleosis, mycoplasma pneumonia, cytomegalovirus and varicella zoster. $[11,12,14]$. Solid tumors including: pulmonary squamous cell carcinoma, metastatic colonic adenocarcinoma, metastatic adrenal adenocarcinoma, basal cell carcinoma may also be associated with secondary CAD [12]

The clinical course of the disease is dependent on the underlying cause. While CAD secondary to lymphoproliferative diseases may present with chronic course and mild hemolysis, a prompt and transient diseases is mostly observed with infectious diseases [12].

PCH is caused by polyclonal IgG type antibodies that bind to red blood cell surface antigens and cause complement-mediated hemolysis. It is a rare disease mostly affects children. The presentation of the disease is acute or chronic. The acute form is mostly observed in children and associated with infections. PCH can also be a side effect of some vaccinations. Chronic idiopathic form PCH also occurs, but it is much more rare [12].

\section{Diagnosis}

The diagnosis is mainly based on anemia mostly macrocytic or normocytic together with reticulocytosis. But, it must be kept in mind that autoantibodies directed against early erythroblasts may also cause reticulocytopenia and severe anemia in certain cases. Low serum haptoglobin, elevated Lactate Dehydrogenase (LDH), increased indirect bilirubin levels, and a positive direct anti-globulin test are other hallmarks of AIHAs. The antiglobulin test or direct coombs test can detect the complements and autoantibodies on the surface of the erythrocytes. At the polyspecific step, both IgG type autoantibodies and complements can be detected by antihuman antiglobulin $\mathrm{G}$ and anticomplement reagents. At the next step, monospecific reagents are used to detect anti- C3d and IgG separately. The presence of only C3d may indicate IgM type antibody $[15,16]$.

The diagnostic workup should also be focused on differential diagnosis of primary or secondary and cold or warm types since treatment and prognosis are completely different in these entities. A careful history including drugs, clues for recent infectious diseases, autoimmune diseases or malignancies and physical examination is crucial especially to rule out secondary causes. In certain circumstances more sophisticated workup with autoantibody profiles including antinuclear or antiphospholipid antibodies, imaging techniques with computed tomography of thorax and abdomen and biopsies including bone marrow biopsy and aspiration may be required to rule out secondary causes $[11,17]$.

\section{Treatment Strategies}

\section{General aspects on the treatment of secondary AIHA}

The treatment of the underlying cause is essential in the secondary forms and withdrawal of the offending agent should also be considered in all drug induced AIHAs. In the drug induced AIHA, a positive DAT test without an evident anemia and hemolysis is not an indication for discontinuation of the offending drug since DAT positivity can be observed without any hemolysis. In most of the cases discontinuation of the drug may ameliorate the hemolysis but in some severe cases steroids should be considered. Red blood cell transfusions can be used in severe and symptomatic cases, although the transfused cells are also at risk of premature destruction [12].

For the management of AIHAs secondary to solid tumors, the curative treatment of the underlying cancer by surgery, chemotherapy or radiotherapy may control the hemolysis. In certain cases steroid may have some benefit although steroid refractoriness may also be observed in some patients with solid tumors [18].

Steroids are the first choice in the patients with CLL who are not candidates for chemotherapy according to stage of the disease. Rituximab alone or rituximab based chemotherapies are alternatives in refractory patients [19]. Although fludarabine is a promising agent in CLL patients, the risk of drug induced hemolysis with fludarabine limits its use in CLL patients with AIHA [20]. In case of B cell lymphomas, treatment of the underlying malignancy should be considered promptly especially with rituximab based chemotherapies [21].

Autoimmune diseases especially SLE can be associated with AIHA. Corticosteroids are the first choice in SLE patients with a high remission induction rate. In relapsed or refractory cases, 
immunosuppressive agents, danazol or rituximab can be tried although there is not a consensus for the treatment of relapsed refractory cases. Although rituximab is a promising agent as a second line treatment in SLE patients with AIHA, the safety and efficacy should be documented with prospective randomized studies [22-24].

\section{Treatment of primary CAD}

Primary strategy for the CAD is to avoid from cold and warm clothing (Table 2). In the case of blood transfusion, blood warmers and keeping the transfused extremity warm should be considered. Since the complement proteins may aggravate hemolysis, blood products with low plasma content are recommended [25-27].

Treatment of asymptomatic patients is not mostly necessary. Steroids are ineffective and not recommended in primary cases $[28,29]$. Since the complement coated red blood cells are mainly cleared from the plasma by liver, splenectomy is an ineffective treatment modality in these patients except ones with IgG type immunoglobulin instead of IgM [28,29].

The preferred treatment in primary CAD patients is rituximab as a combination or monotherapy. Berentsen et al, treated 27 CAD patients with 37 courses of hemolysis with 4 courses of rituximab in a week apart and obtained a favorable response rate [30]. Fludarabine combination with rituximab is another option in symptomatic patients, even patients refractory to rituximab monotherapy. Grade 1-3 cytopenias related to chemotherapy were asymptomatic and the treatment was accepted as well tolerated. Since CAD is mostly observed in elderly patients, the risk benefit ratio should be assessed carefully to avoid excess toxicity of fludarabine [31].

Other promising agents are proteosome inhibitor bortezomib, an anti-C5 antibody eculuzimab, and bendamustin [29,32,33]. In the literature the effectiveness and safety of these agents were reported as case reports. Since CAD is a rare disease, performing a randomized controlled trials is difficult, so evidence based data is needed to establish the treatment of first line and the refractory patients in CAD.

\section{Treatment of Paroxysmal cold hemoglubinuria}

PCH is a subtype of AIHA occurred due to cold autoantibodies of

Table 2: Treatment of primary CAD:

1. Supportive care

a. Avoid cold, warm clothing

b. Transfusion with low plasma content blood products

c. Blood warmers

d. Keeping transfused extremity warm

2. First line treatment:

Rituximab: monotherapy or in combination

3. Secondline treatment*:

Eculizumab

Bortezomib

Bendamustine

CAD : cold agglutinin disease

*: in refractory cases, there is not strong evidence in the literature
IgG type. The prognosis of the disease is good although fatal cases were reported [34]. Although patients may require supportive care and red blood cell transfusions, the rate of hemolysis will decrease due to the transient nature of the disease [34]. Steroid is an alternative in patients with treatment indication even though the effectiveness of the immunosuppressive treatment is not well documented.

\section{Treatment of primary warm AIHA}

General aspects: Since WAIHA is rare and heterogeneous disease, randomized controlled phase 3 trials are lacking. The guidelines in the literature are mainly based on case reports, small case series and a few prospective studies [35,36]. So, the treatment decision should be made on an individual basis. The aim of the treatment is to control the hemolytic crisis and anemia to provide patient comfort and maintain quality of life. Since there is chronic course in most of the cases, prevention of the side effects of the treatment has a priority [35].

One of the most important decision in acute WAIHA is requirement for immediate transfusion. This decision should be made according to patient characteristics, the severity of the anemia, and other comorbidities of the patients.

Since the autoantibodies are against blood group antigens, cross matched compatible red blood cell transfusion is nearly impossible. Under these circumstances, in severely anemic symptomatic patients incompatibility is not a contraindication for transfusion. All the effort should be performed to exclude alloantibodies in risk groups before transfusion [35,36]. In vivo compatibility tests can be also performed by giving $20 \mathrm{ml}$ of donor blood and followed by observation of any side effect in very limited cases [35]. While red blood cell transfusion is encouraged in severely ill and symptomatic patients, large amount of uncontrolled transfusion should be restricted [37].

First line treatment: In the primary AIHA, the treatment of choice is prednisone or methylprednisolone at a dose of $1 \mathrm{mg} /$ $\mathrm{kg}$ per day as a first line therapy [35-38]. Although higher doses can be used, there is not sufficient data supporting additional benefit of higher doses [38]. Treatment with the starting dose can be continued for 3 weeks. If there is no response after 3 weeks, second line treatment options should be reviewed [35]. After a hemoglobin level of $10 \mathrm{gr} / \mathrm{dl}$ is achieved, tapering of the dose should be considered. Although there is not a consensus for the total duration of the steroid treatment, the tapering of the dose should be made slowly [35]. Treatment with a low dose can be continued for 3 months after a response is achieved since relapses are frequent after cessation of the drug [35,37]. Supportive care during steroid treatment with vitamin D, calcium preparations, and folic acid should be kept in mind in all patients [35].

The remission with steroid is high and rapid in most of the patients. In a retrospective study consisting of 308 primary AIHA patients, the remission rate with steroid treatment is as high as $80 \%$ in warm Ig G type AIHA [36]. Beside this high rate of remission, steroid dependence and relapses after cessation of the disease is also common. In these refractory patients, IV 
immunoglobulins can be administered [36,37]. Danazol, an androgen analogue is another alternative agent that can be used as a steroid sparing agent in first line treatment. In a retrospective study, Pignon, et al., reported that addition of danazol to steroid in the first line treatment may decrease the total duration of steroid therapy. Danazol can also be used in patients who are refractory to steroids with an acceptable side effect profile [39].

Second line treatment: Although steroid provides high rates of remission in wAIHA, steroid dependence, steroid refractoriness and relapses after cessation of the drug still constitutes a problem in clinical settings. Approximately $15 \%$ of the patients need higher maintenance doses to provide an acceptable hemoglobin levels. In the long term, steroid side effects predominate and overweight the positive effects of steroid. Patients who have stable and acceptable hemoglobin levels under a dose of $0.1 \mathrm{mg} /$ $\mathrm{kg} /$ day may be treated with long term low dose steroid [35].

In refractory or patients who need higher doses of steroid should be assessed for second line treatment [35]. Several options are available in steroid refractory patients.

Splenectomy: Splenectomy is an option in steroid refractory patients in whom operation is not contraindicated. But exact time for the operation is not well documented $[35,37,40]$. Coon W. evaluated splenectomy in 52 autoimmune hemolytic anemias. Steroids were discontinued in $64 \%$ of the patients and steroid doses could be reduced to acceptable doses in $21 \%$ of the patients. Although randomized controlled studies are lacking in the literature, splenectomy provides a short term efficacy and good initial response in refractory or steroid dependent patients $[35,37,40]$.

The splenectomy can be performed laparoscopically in most of the cases with a low perioperative mortality [35]. Postoperative intraabdominal bleeding, intraabdominal infections or wound infections and abdominal wall hematomas are observed in a low incidence especially after improvements in surgical procedures and post-operative care. One of the most frightening complication is sepsis with encapsulated microorganisms, pneumococci, meningococci, and hemophilus. The risk of infection is low but the mortality rate of the infections is high so vaccination against mentioned microorganisms is recommended before the operation $[35,41,42]$. The risk of post-operative thromboembolism and pulmonary hypertension should not be overlooked in patients with splenectomy. Under these circumstances, the decision should be made by individual basis by considering the risk and benefit of the operation. It should also be kept in mind that splenectomy is the only treatment option that provide a freedom from treatment in about one in five patients for long periods [35].

Rituximab: The data of rituximab is mainly based on small prospective and retrospective studies. The recommended dose is $375 \mathrm{mg} / \mathrm{m}^{2}$ in every week for 4 weeks. A retrospective analysis consisted of 11 primary wAIHA patients who were with rituximab revealed an overall response rate of $64 \%$ [43]. Another study by Bussone, et al., demonstrated a 93\% of overall initial response to rituximab therapy with standard doses [44]. Relapse is unavoidable during follow up periods but retreatment with rituximab is effective in these patients $[44,45]$. Since patients may benefit from second course of rituximab, intermittent or a maintenance therapy should be considered. But this data should be confirmed in prospective randomized studies.

Although most of the studies include heterogeneous group of patients and are retrospective, collected data supports that, rituximab is an effective therapy with an acceptable side effect profile. It is the best option in steroid refractory patients especially who are not candidate for splenectomy (Table 3). The most important contraindication of rituximab is untreated hepatitis B infection which should be kept in mind [35].

A prospective study was conducted for the efficacy and safety of the rituximab as a first line treatment. In this study, 64 newly diagnosed WAIHA patients were treated either with steroid monotherapy or with combination of rituximab and steroid. The response rate and relapse free survival rates were statistically better in the combination group [46].

A phase 3 multicenter randomized double blind and placebo controlled study supported that rituximab combined with prednisone may be effective and safe as a first line therapy. In this phase 3 trial, 27 patients were followed for at least 1 year. The overall response rate at 1 year was $75 \%$. After 2 years, 10/16 patients with rituximab versus $3 / 16$ with placebo still showed CR $(P=0.011)$. There is no statistical difference between the two groups in terms of side effects [47].

Low dose rituximab, $100 \mathrm{mg} /$ weekly for 4 weeks, is another alternative with low cost and possible lower rate of side effects. In the retrospective trial of 49 patients, effectiveness and safety of low dose rituximab combined with steroid was analyzed [48]. It was demonstrated that rituximab in lower doses could inhibit antibody production in in vitro studies, which is compatible with its effectiveness and safety in clinical settings [49] .

Table 3: Treatment of primary warm AIH:

A.First line

Steroid $1 \mathrm{mg} / \mathrm{kg}$

Together with

Ca preperation

Folic acid

Vitamin D

B.Second line

1.Splenectomy

2.Rituximab (especially in patients who are not candidate for operation)

C.Third line

Immunsupresive agents

Azothioprine

Cyclophosphomide

Cyclosporine

Mycophenolate mofetil

D.In very selected

1.High dose cyclophosphomide

2.Alemtuzumab

3. Hematopoetic stem cell transplantation 


\section{Third line treatment}

Immunosuppressive agents: Treatment choices and response rates decreases in patients who are refractory to both rituximab and splenectomy. Immunosuppressive agents are alternatives in this group. Azothioprine with a dose of 100-150 $\mathrm{mg} /$ day or cyclophosphomide $100 \mathrm{mg} /$ day is an option in these patients as third line treatment [35]. Although they are effective in a group of patients, the overall response rates are not well documented [35,41]. Cyclosporin and mycophenolate mofetil are also used in very limited number of patients [50-52]. Although the results are promising, selection biases, doubts about efficacies and the side effects of these drugs should be considered before initiation of therapy.

Other therapies: Alemtuzumab, a monoclonal antibody against CD 52 is an alternative in very sellected patients. In cases refractory to all alternative strategies, alemtuzumab is an option. It should be used with causion since it may cause severe lymphopenia and oppurtunistic infections including activation of cytomegalovirus $[10,53]$. Very limited number of refractory and transfusion dependent patients may benefit from allogeneic stem cell transplantation. Since mortality and morbidity rates are high with transplantation, benefit risk ratio should be evaluated carefully [10].

As a conclusion, AIHA is a heterogeneous disease. Although there are effective therapies, refractoriness or relaspses are still a problem. In this context, the pathogenesis becomes important. Although it is known that complement system, macrophages, $\mathrm{T}$ cell lymphocytes and cytokines play an important role in the pathogenesis, the relative burden of these mechanisms or immunologic pathways are not well documented. Specific pathways inhibitors, new monoclonal antibodies directed against $\mathrm{B}$ and T cells, complemet system inhibitors or immunomodulatory drugs are promising agents in especailly refractory cases. Further understanding of the disease and its heterogenecity will give insights for developing new drugs.

\section{References}

1. Barcellini W. NewInsights in the Pathogenesis of Autoimmune Hemolytic Anemia. Transfus Med He mother. 2015;42(5):287-293. Doi:10.1159/000439002

2. Okada S, Miyoshi Y, Takizawa Y, et al. Fulminant autoimmune hemolytic anemia with multiple organ failure. Rinsho Ketsueki. 1989;30(10):1892-1897

3. Gomard-Mennesson E, Ruivard M, Koenig M etal. Treatment of isolated severe immune hemolytic anaemia associated with systemic lupus erythematosus : 26 cases. Lupus. 2006;15(4):223-231.

4. Hauswirth AW, Skrabs C, SchutzingerC et al. Autoimmune hemolytic anemias, Evans'syndromes, and pure red cell aplasia in non-Hodgkin lymphomas. Leuk Lymphoma. 200748:1139-1149.

5. Atta M, Brannigan ET, Bain BJ. Cold autoimmune hemolytic anemia secondary to a typical pneumonia. Am J Hematol. 2017;92(1):109. Doi:10.1002/ajh.24550

6. Eskazan AE, Dal MS, Kaya S, Dal T, Ayyildiz O, Soysal T. Two cases of autoimmune hemolytic anemia secondary tobrucellosis:a review of hemolytic disorders in patients with brucellosis. InternMed. 2014;53(11):1153-1158.
7. Shah MB, Nanjapp V, Devaraj HS, Sindhu KS Autoimmune hemolytic anaemia in Hodgkin's lymphoma. J Assoc Physicians India. 2013;61(7):492-494.

8. Garratty G, Arndt PA. Drugs that have been shown to cause druginduced immune hemolytic anemia or positive direct antiglobulin tests:some interesting findings since 2007 Immunohematology. 2014;30(2):66-79.

9. Vehapoğlu A, Göknar N, Tuna R, Çakır FB. Ceftriaxone-induced hemolytic anemia in a child successfully managed with intravenous immunoglobulin. Turk J Pediatr. 2016;58(2):216-219.

10. Kalfa TA. Warm antibody autoimmune hemolytic anemia. Hematology Am Soc Hematol Educ Program. 2016;2(1):690-697. DOI:10.1182/ asheducation-2016.1.690

11. Bass GF, Tuscano ET, Tuscano JM Diagnosis and classification of autoimmune hemolytic anemia. Autoimmun Rev. 2014;13(4-5):560564. Doi:10.1016/j.autrev.2013.11.010

12.Gehrs BC, Friedberg RC.Autoimmune hemolytic anemia. Am J Hematol.2002 Apr;69(4):258-271.

13. Sokol RJ, Hewitt S, Stamps BK. Autoimmune haemolysis: an 18-year study of 865 cases referred to a regional transfusion centre. Br Med J (ClinResEd) 1981;282(6281):2023-2027.

14. Barcia Ruiz JM, García Díez I, Fernándezde La Mata M, Mascort Vaca G, Valdivia Bautista L, Plata Rosales JC. Autoimmune hemolytic anemia secondary to cytomegalovirus infection. An Esp Pediatr. 2002;57(3):268-269.

15. Barcellini W, Fattizzo B. Clinical Applications of Hemolytic Markers in the Differential Diagnosis and Management of Hemolytic Anemia. Dis Markers. 2015;2015:635670. doi:10.1155/2015/635670

16. Zantek ND, Koepsell SA, Tharp DR Jr, Cohn CS. The direct antiglobulin test: a critical step in the evaluation of hemolysis. Am J Hematol. 2012;87(7):707-709. Doi:10.1002/ajh.23218

17. Lechner K, Jäger U. How I treatautoimmune hemolytic anemias in adults. Blood. 2010;116:1831-1838. Doi:10.1182/blood-2010-03-259325

18. Puthenparambil J, Lechner K, Kornek G. Autoimmune hemolytic anemia as a paraneoplastic phenomenon in solid tumors: A critical analysis of 52 cases reported in the literature. Wien KlinWochens chr. 2010;122(7-8):229-236. Doi:10.1007/s00508-010-1319-z

19. Rossignol J, Michallet AS, Oberic L et al. Rituximab-cyclophosphamidedexamethasone combination in the management of autoimmune cytopenias associated with chronic lymphocytic leukemia. Leukemia. 2011;25(3):473-478. Doi:10.1038/leu.2010.278

20. Nishida H, Murase T, Ueno H, Park JW, Yano T, Ikeda Y. Fludarabineassociated autoimmune hemolytic anemia occurring in B-cell chronic lymphocytic leukemia. Leuk Res.2006;30(12):1589-1590.

21. Hauswirth AW, Skrabs C, Schützinger C, Gaiger A, Lechner K,Jager U.Autoimmune hemolytic anemias,Evans'syndromes,and pure red cell aplasia in non-Hodgkin lymphomas.LeukLymphoma.2007;48(6):1139-1149.

22. Gomard-Mennesson E, Ruivard M, Koenig M et al. Treatment of isolated severe immune hemolytic anaemia associated with systemic lupus erythematosus:26 cases. Lupus. 2006;15(4):223-231.

23. Fayyaz A, Igoe A, Kurien BT et al. Haematological manifestations of lupus. Lupus Sci Med. 2015;2(1):e000078. Doi:10.1136/ lupus-2014-000078 
24. Scheinberg M, Hamerschlak N, Kutner JM etal. Rituximab in refractory autoimmune diseases : Brazilian experience with 29 patients (20022004). Clin Exp Rheumatol. 2006;24(1):65-69.

25. Berentsen S. How I manage cold agglutinin disease. Br J Haematol 2011;153(3):309-317. DOI:10.1111/j.1365-2141.2011.08643.x

26. Arthold C, Skrabs C, Mitterbauer-Hohendanner G et al. Cold antibody autoimmune hemolytic anemia and lymphoproliferative disorders: a retrospective study of 20 patients including clinical, hematological, and molecular findings. Wien Klin Wochenschr. 2014;126(1112):376-382. doi:10.1007/s00508-014-0547-z

27. Swiecicki PL, Hegerova LT, Gertz MA. Cold agglutinin disease. Blood. 2013 122(7):1114-1121. doi:10.1182/blood2013-02-474437

28. Berentsen S, Ulvestad E, Langholm R, et al. Primary chronic cold agglutinin disease:a population based clinical study of 86 patients. Haematologica. 2006;91(4):460-466.

29. Berentsen S. Cold agglutinin disease. Hematology Am Soc Hematol Educ Program. 2016;2(1):226-231. DOI:10.1182/asheducation-2016.1.226

30. Berentsen S, Ulvestad E, Gjertsen BT. Rituximab for primary chronic cold agglutinin disease:a prospective study of 37 courses of therapy in 27 patients. Blood. 2004;103(8):2925-2928. DOI:10.1182/ blood-2003-10-3597

31. Berentsen S, Randen U, Vågan AM, et al. High response rate and durable remissions following fludarabine and rituximab combination therapy for chronic cold agglutinin disease. Blood. 2010;116(17):3180-3184 Doi:10.1182/blood-2010-06-288647

32. Carson KR, Beckwith LG, Mehta J. Successful treatment of Ig M mediated autoimmune hemolytic anemia with bortezomib. Blood. 2010;115(4):915. Doi:10.1182/blood-2009-09-242917

33. Gupta N, Wang ES. Long-term response of refractory primary cold agglutinin disease to eculizumab therapy. AnnHematol. 2014Feb;93(2):343-344. Doi:10.1007/s00277-013-1800-7

34. Sokol R.J., Hewitt S., Stamps B.K. Auto immune haemolysis associated with Donath-Landsteiner antibodies. Acta Haematol. 1982;68(4):268277.

35. Lechner K, Jäger U. How I treat autoimmune hemolytic anemias in adults. Blood. 2010;116(11):1831-1838. Doi:10.1182/ blood-2010-03-259325

36. Barcellini W, Fattizzo B, Zaninoni A. ical heterogeneity and predictors of outcome in primary autoimmune hemolyticanemia:a GIMEMA study of 308 patients. Blood. 2014;124(19):2930-2936. Doi:10.1182/blood-2014-06-583021

37. Michel M. Warm auto immune hemolytic anemia: advances in pathophysiology and treatment. Presse Med. 2014;43(4 Pt 2):e97-e104. doi:10.1016/j.lpm.2014.02.009

38. Crowther M, Chan YL, Garbett IK, Lim W, Vickers MA, Crowther MA. Evidence-based focused review of the treatment of idiopathic warm immune hemolytic anemia in adults. Blood. 2011;118(15):4036-4040. Doi:10.1182/blood-2011-05-347708
39. Pignon JM, Poirson E, Rochant H. Danazol in autoimmune haemolytic anaemia. Br J Haematol. 1993;83(2):343-345.

40. Coon WW. Splenectomy in the treatment of hemolytic anemia. ArchSurg. 1985;120(5):625-628.

41.Zanella A, Barcellini W. Treatment of autoimmune hemolytic anemias. Haematologica. 2014;99(10):1547-1554. Doi:10.3324/ haematol.2014.114561

42. Bisharat N, Omari H, Lavi I, Raz R. Risk of infection and death among post-splenectomy patients. J Infect. 2001;43(3):182-186.

43. D'Arena G, Califano C, Annunziata M, et al. Rituximab for warm-type idiopathic autoimmune hemolytic anemia: a retrospective study of 11 adult patients. Eur J Haematol. 2007;79(1):53-58.

44. Bussone G, Ribeiro E, Dechartres A et al. Efficacy and safety of rituximab in adults warm antibody autoimmune haemolytic anemia: retrospective analysis of 27 cases. Am J Hematol. 2009;84(3):153157. Doi:10.1002/ajh.21341

45. Maung SW, Leahy M, O'Leary HM et al. A multi-centre retrospective study of rituximab use in the treatment of relapsed or resistant warm autoimmune haemolytic anaemia. Br J Haematol. 2013;163(1):118122. Doi:10.1111/bjh.12486

46. Birgens H, Frederiksen H, Hasselbalch HC. A phase III randomized trial comparing glucocorticoid monotherapy versus glucocorticoid and rituximab in patients with autoimmune haemolytic anaemia. Br J Haematol. 2013;163(3):393-399. Doi:10.1111/bjh.12541

47. Michel M, Terriou L, Roudot-Thoraval F et al. A randomized and double blind controlled trial evaluating the safety and efficacy of rituximab fo rwarm autoimmune hemolytic anemia in adults (the RAIHA study). Am J Hematol. 2017;92(1):23-27.doi:10.1002/ajh.24570

48. Fu R, Yan S, Wang X et al. A monocentric retrospective study comparing pulse cyclophosphamide therapy versus low dose rituximab in the treatment of refractory autoimmune hemolytic anemia in adults. Int J Hematol. 2016;104(4):462-467. Doi:10.1007/s12185-016-2056-5

49. Barcellini W, Zaja F, Zaninoni A et al. Sustained response to low-dose rituximab in idiopathic autoimmune hemolytic. 2013;91(6):546-551. Doi:10.1111/ejh.12199

50. Howard J, Hoffbrand AV, Prentice HG, Mehta A. Mycophenolate mofetil for the treatment of refractory auto-immune haemolytic anemia and auto-immune thrombocytopenic purpura. Br J Haematol. 2002;117:712-715.

51. Kotb R, Pinganaud C, Trichet C, et al. Efficacy of mycophenolate mofetil in adult refractory auto-immune cytopenias:a single center preliminary study. Eur J Haematol. 2005;75(1):60-64.

52. Emilia G, Messora C, Longo G, Bertesi M. Long-term salvage treatment by cyclosporin in refractory autoimmune haematological disorders. Br J Haematol.1996;93:341-344.

53. Jaime-Pérez JC, Rodríguez-Martínez M, Gómez-de-León A, TarínArzaga L, Gómez-Almaguer D. Current approaches for the treatment of autoimmune hemolytic anemia. Arch Immunol Ther Exp (Warsz). 2013;61(5):385-395. doi:10.1007/s00005-013-0232-3 